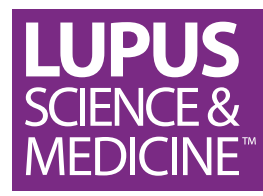

To cite: Wang $\mathrm{H}, \mathrm{GaO} \mathrm{Y}$, Ma Y, et al. Performance of the 2019 EULAR/ACR systemic lupus erythematosus classification criteria in a cohort of patients with biopsyconfirmed lupus nephritis. Lupus Science \& Medicine 2021;8:e000458. doi:10.1136/ lupus-2020-000458

- Additional material is published online only. To view, please visit the journal online (http://dx.doi.org/10.1136/ lupus-2020-000458).

Received 5 November 2020 Revised 6 January 2021 Accepted 9 January 2021

A) Check for updates

(C) Author(s) (or their employer(s)) 2021. Re-use permitted under CC BY-NC. No commercial re-use. See rights and permissions. Published by BMJ.

Kidney Disease Center, Zhejiang University School of Medicine First Affiliated Hospital; Institute of Nephrology, Zhejiang University; Key Laboratory of Kidney Disease Prevention and Control Technology, Hangzhou, Zhejiang, China

Correspondence to Dr Fei Han; hanf8876@zju. edu.cn

\title{
Performance of the 2019 EULAR/ACR systemic lupus erythematosus classification criteria in a cohort of patients with biopsy-confirmed lupus nephritis
}

Huijing Wang, Yunjie Gao, Yanhong Ma, Fanghao Cai, Xiaohan Huang, Lan Lan, Pingping Ren, Yaomin Wang, Jianghua Chen, Fei Han

\section{ABSTRACT}

Objective To evaluate the performance of the European League Against Rheumatism/American College of Rheumatology (EULAR/ACR) SLE classification criteria in a cohort of patients with biopsy-confirmed lupus nephritis (LN) and their renal prognosis.

Methods Patients with newly diagnosed SLE attending and followed up for $>12$ months were included. A retrospective review of all patients with renal biopsy fulfilling a consensus expert opinion during 2014 and 2018. Clinical, serological and pathological data were collected and each patient was assigned a high/low criteria scores (HS/LS) group. Survival curves for flare adjusted for multiplicity on renal flares, was applied to the two groups.

Results Applying EULAR/ACR criteria in our cohort of 126 patients, 6 (4.76\%) did not meet the criterion, resulting in a sensitivity of $95.24 \%$. The EULAR/ACR criteria scores was positively correlated with SLE disease activity index scores. Additionally, we noticed that a significant difference in clinical and immunological manifestations between HS and LS group. We observed a higher proportions of class III or IV LN and lower proportions of class II or V LN ( $p=0.034)$ and pathological higher activity index in HS group ( $p=0.007)$. Compared with LS groups, patients involved more severe renal damage and achieved higher rate of complete remission in the HS group. The Kaplan-Meier exploratory analyses, adjusted for LN classification, estimated glomerular filtration rate, activity index and chronicity index and induction and maintenance treatments, showed that patients in the HS group had a tendency of higher renal flare risk than that in the LS group ( $H R=0.21, p=0.04)$.

Conclusions The EULAR/ACR criteria performed high sensitivity in identifying SLE in this cohort of biopsyconfirmed LN. Patients with LN with high criteria scores had more extrarenal manifestations, and worse renal prognosis in the short and long terms.

\section{INTRODUCTION}

SLE is a systemic autoimmune disease with various clinical manifestations in multiple

\section{Key messages}

What is already known about this subject?

- The 2019 European League Against Rheumatism/ American College of Rheumatology (EULAR/ACR) SLE classification criteria provided differential classification for SLE with high sensitivity and specificity in patients with ANA $\geq 1: 80$ as an entry criterion.

What does this study add?

- The 2019 EULAR/ACR criteria showed a quite high sensitivity in biopsy-confirmed patients with LN.

- The biopsy-proven patients with LN with high EULAR/ ACR criteria scores had more extrarenal manifestations, and a tendency of higher renal flare.

How might this impact on clinical practice or future developments?

- Patients with LN with high new criteria score are at high risk of renal flares and need more close monitoring in long term.

organs including kidney damage. ${ }^{1}$ SLE classification criteria have traditionally been focused on by rheumatology clinics rather than non-rheumatology clinics. Particularly, this may be relevant to nephrologists, because patients with renal biopsy findings reminiscent of lupus nephritis (LN) would readily be evaluated based on the criteria to determine the classification. ${ }^{2}$ Remarkably, the definition of biopsy-confirmed LN needs to be specified in both SLE classification and histopathological classification. Therefore, the Systemic Lupus International Collaborating Clinics (SLICC) 2012 classification contains additional cases with 'renal lupus only' provided they are biopsy proven together with detectable ANA and/or anti-Smith antibodies and/or anti-double-stranded (ds)DNA antibodies. ${ }^{34}$ SLICC 2012 classification addressed 
the importance of valid SLE classification criteria in the nephrology clinic by increasing the weight of renal lupus to help distinguish patients with $\mathrm{LN}$ better.

The recently developed European League Against Rheumatism/American College of Rheumatology (EULAR/ACR) 2019 criteria set was noted to perform more excellently than previous SLE classification criteria including the 1997 revised ACR SLE classification criteria and 2012 SLICG classification criteria. ${ }^{56}$ This classification criteria renewed two novel concepts, namely ANA as an entry criterion along with variably weighed features including seven clinical domains and three immunological items. A total score of 10 points is considered as a cut-off for SLE classification. And these new criteria still reserve the earlier classification of LN based mainly on kidney biopsy. If the entry criterion is fulfilled, for the patients with renal biopsy, they are classified into SLE when they met the criteria of biopsy-proven class III or IV LN or biopsy-proven class II or V LN with at least one more criterion.

As reported recently, the weighted criteria also could correlate positively with the disease activity of SLE by SLE disease activity index (SLEDAI) ${ }^{78}$ In light of the alteration in the EULAR/ACR 2019 classification and the unknown performance of the classification in patients from nephrology clinics, we aimed to evaluate the validity of EULAR/ACR classification in a cohort of patients based on a biopsy feature characteristic of LN. From the perspective of nephropathology, the definition of biopsyconfirmed LN with a 'full house' pattern of immunofluorescence would certainly raise the clinicopathological possibility of SLE as a differential classification criterion. ${ }^{2}$ In this study, we assessed the performance of the EULAR/ ACR compared with the SLICC classification criteria to distinguish patients with SLE with biopsy-confirmed LN. Moreover, we retrospectively analysed the clinical data and biopsy findings of patients with LN based on EULAR/ ACR criteria scores in this cohort.

\section{MATERIALS AND METHODS}

\section{Study design and patients}

This is a single-center, retrospective study. The study population included 126 patients diagnosed with LN based on renal biopsy-proven 2003 International Society of Nephrology/Renal Pathology Society (ISN/RPS) LN classification $^{9}$ between January 2014 and December 2018, and were followed up for $>12$ months at the Kidney Disease Center of First Affiliated Hospital of Zhejiang University School of Medicine. Patients with biopsy-confirmed LN were reviewed for the 2012 SLICC and the 2019 EULAR/ ACR classification criteria, starting at the visit with the diagnosis of LN. Notably, 2012 SLICG criteria including immunological items of a positive ANA (above laboratory reference range), while 2019 EULAR/ACR criteria mandate the entry criterion of a positive ANA titre of $\geq 1: 80$ on HEp-2 cells.

\section{Demographic and clinical data}

Baseline demographic and clinical data were obtained from the electronic medical records of our hospital. Baseline clinical examination included serum ANA antibodies, anti-dsDNA antibodies, anti-Smith antibodies, anti- $\beta 2 \mathrm{GP} 1$ antibodies, white blood cell count, red blood cell count, platelet count, serum albumin, serum creatinine $(\mathrm{SCr})$, C3 and C4. For ANA detecting, ANA immunofluorescence on HEp-2 cells were performed using kits (Euroimmun, Germany). The cut-off for ANA of our laboratory positivity was 1:40, as manufacturer recommended. And patients with ANA at a titre of $\geq 1: 80$ in our lab were recognised as fulfilment of the entry criteria of the 2019 EULAR/ACR classification. Urine protein creatinine ratio (UPCR) was tested in substitution of 24-hour urine protein. SLEDAI scores were assessed and clinical symptoms and manifestations were recorded when the patients were hospitalised and confirmed by a consensus expert opinion.

\section{Pathological data}

One pathologist reported the following pathological features based on ISN/RPS criteria and another pathologist reviewed and confirmed these results of renal biopsies when we did our current study. Both glomerular sclerosis and crescents (cellular, fibrous, fibrocellular and segmental) were calculated and described as percentage. In addition, histological features such as mesangial proliferation, interstitial inflammation were present with the degree of severity (mild, moderate and severe). And the immunofluorescence for deposition of IgM, IgG, IgA and complement factors C3, C4 and C1q were measured with a semi-quantitative scoring system, where $0,1,2$ and 3 correspond to the degree of severity (absent, mild, moderate and severe, respectively). Other lesions, such as capillary tuft necrosis was displayed as $0 / 1$ in terms of their existence. Activity index (AI) and chronicity index (CI) were present as mean $\pm \mathrm{SD}$.

\section{Definition of remission and flare}

Treatment response included complete remission, partial remission and treatment failure on basis of previous study. ${ }^{10-14}$ Complete remission requires $\mathrm{uPCR}<0.5 \mathrm{~g} / \mathrm{g}$, inactive urinary sediments, normal serum albumin and creatinine concentration. Urine sediment was considered inactive in the absence of red blood cell casts, white blood cell casts and glomerular haematuria $(<5 \%$ dysmorphic red blood cells per high power field). Partial remission was defined as stabilisation $( \pm 25 \%)$, or improvement of SCr, but not to normal, plus a $\geq 50 \%$ decrease in uPCR by 6 months. ${ }^{12}$ Treatment failure was defined as a sustained $25 \%$ increase in SCr $>3$ months and failure to meet the urinary protein excretion standard for partial remission for longer than 6 months.

Flare including nephritic relapse and proteinuric relapse was defined as an increase in disease activity that required restarting immunosuppression. ${ }^{11} 14$ A nephritic relapse indicated a recent increase of $\mathrm{SCr}$ by $25 \%$ with 
active urinary sediments. A proteinuric relapse suggests a persistent raise of proteinuria either $>1 \mathrm{~g} / \mathrm{d}$ after complete remission, or $>2 \mathrm{~g} /$ day and twice the previous value after partial remission. We used the first occurrence of either end-stage renal disease (ESRD) or death as a long-term outcome referred to Kidney Disease: Improving Global Outcomes (KDIGO) guidelines. ${ }^{15}$

\section{Statistical analysis}

All the analyses were conducted by SPSS software (V.22.0) and GraphPad (V.5.0). For normally distributed variables (means $\pm \mathrm{SD}$ ), the association between groups was analysed by Student's t-test. The Mann-Whitney U test was used for those with non-normal distribution (medians). Categorical data were compared using $\chi^{2}$ test and Fisher's exact test. The Pearson's correlation test was used for the analysis of SLEDAI score and 2019 criteria scores. The sensitivity of SLICC and EULAR/ACR classifications were assessed. Kaplan-Meier curve was applied to the two groups, and renal flare-free survival was adjusted for the several confounders by Cox regression analysis. P value $<0.05$ was used to define significance. Otherwise, the important renal-related determinants with significance in the univariate analysis were included in the Cox regression analysis between the two groups.

\section{RESULTS}

A total of 126 samples with renal biopsies were identified as LN in the nephrology clinic of our hospital between January 2014 and December 2018. Of these 126 patients, 6 cases (non-SLE group) did not meet the 2019 EULAR/ ACR criteria, while the remaining 120 fulfilled these proposed criteria. The total score with the 2019 criteria of patient meeting new criteria ranged from 10 to 42 (mean, 25.1). So, we divided these 120 patients into two groups: high score group (HS group, 2019 criteria scores $>25, \mathrm{n}=59$ ) and low score group (LS group, 2019 criteria scores $\leq 25, n=61$ ). The flow chart of inclusion is shown in online supplemental figure 1 .

\section{Comparison of EULAR/ACR domains of patients based on the EULAR/ACR classification criteria status}

As shown in online supplemental table 1, we compared the baseline clinical characteristics, clinical and immunological manifestations between the non-SLE group and SLE groups based on the 2019 EULAR/ACR classification criteria status. The two groups did not show any significant difference in terms of mean age and sex. Disease activity assessed by the SLEDAI showed no significant differences between the groups either. The mean SLEDAI score was 18.0 for those who did not meet the new SLE criteria and 18.48 for those who did.

As ANA acts as an entry criterion, patients who did not fulfill the criteria had completely different ANA titres than patients who fulfilled the criteria: one had an ANA titre of 1:20, five had an ANA titre of 1:40. Among six patients with the ANA titre of $<1: 80$, one with weak positive anti-Sm antibody and one with weak positive
anti-dsDNA antibody. We then looked at the individual criteria scores within the 2019 EULAR/ACR classification schema. Compared with the group that did not meet the criteria, the total EULAR/ACR criteria mean score in the group that met the criteria was higher, although resulting in no statistically significant difference $(p=0.098)$. Different from Beth Israel Deaconess Medical Center (BIDMC) SLE cohort, ${ }^{16}$ the two groups of patients did not present a statistically significant difference in each individual criteria of clinical manifestation. In view of the immunology domains, highly specific SLE antibodies were noted to be less frequent in the group that did not fulfil the criteria $(\mathrm{p}=0.019)$, although individual anti-Sm or anti-dsDNA antibodies did not differ significantly in the two groups. All but one patient did not have anti-Sm antibodies. Similarly, anti-dsDNA antibodies were not detected in five out of six patients. Altogether, the subjects who met new criteria had a higher incidence of ANA, anti-Sm, anti-dsDNA, compared with the ones who did not.

\section{Performance of the 2019 EULAR/ACR criteria in biopsy- confirmed lupus nephritis}

At the time of renal biopsy, 125 patients in our cohort with clinical SLE fulfilled $\geq 4$ SLICC criteria for the classification of SLE or additional cases with 'renal lupus only' provided they are biopsy-proven LN together with detectable ANA and/or anti-Sm and/or anti-dsDNA antibodies, while six subjects did not meet an entry criterion of 2019 EULAR/ACR criteria. The 2019 EULAR/ACR and 2012 SLICC criteria in our cohort displayed a sensitivity of $95.24 \%$ and $99.20 \%$, respectively. Without the pathological data of renal biopsy, the 2019 criteria scores of seven subjects in the group that met the criteria were $<10$ points. On the basis of the pathological data of renal biopsy, the sensitivities of the new criteria and 2012 SLICC criteria were elevated from $89.68 \%$ to $95.24 \%$, and $90.48 \%$ to $99.20 \%$, respectively (online supplemental figure 2 ). We further explore the relationship between 2019 criteria score and SLEDAI score in our cohort (figure 1). Similar to previous study, ${ }^{7}$ we also found that 2019 criteria score correlated positively with SLEDAI score $(\mathrm{p}<0.0001$, $\mathrm{r}=0.42$ ), suggesting the 2019 criteria may be correlated with SLE disease activity in patients with LN.

\section{Comparison of EULAR/ACR domains in patients with SLE with high and low criteria scores}

We compared the baseline clinical characteristics between the HS group and LS groups based on the EULAR/ACR criteria scores (table 1). The two groups showed no significant difference in terms of mean age, sex and disease duration. Naturally, the HS group showed significantly higher in the mean SLEDAI score and mean EULAR/ ACR criteria score compared with the LS group. It must be noted, however, that class II or V LN were less prevalent and class III or IV LN were more common in the HS group compared with the LS group $(5.25 \%$ vs $39.34 \%$, $84.75 \%$ vs $60.66 \%$, respectively, $\mathrm{p}=0.003)$. Additionally, we 


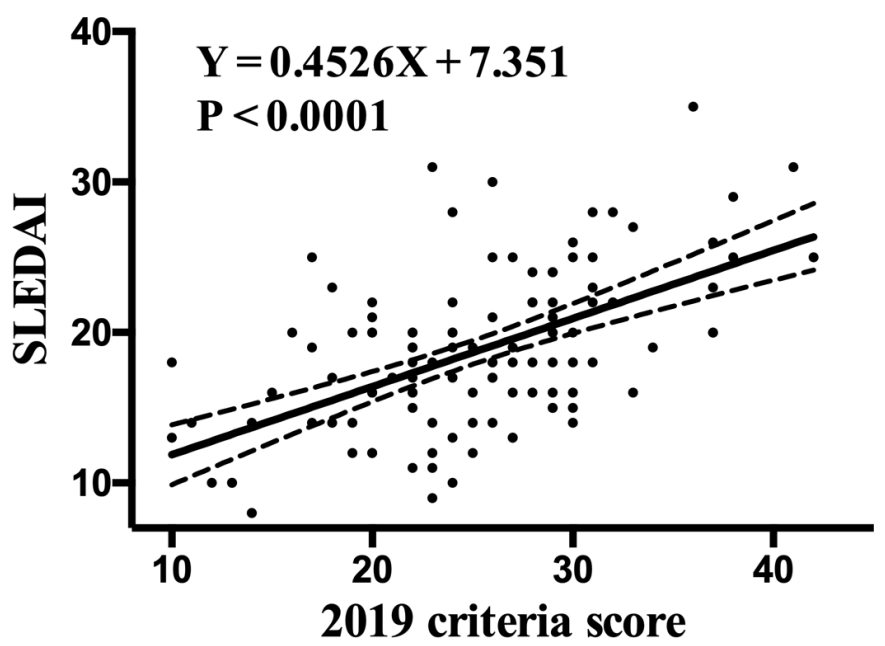

Figure 1 The correlation of 2019 European League Against Rheumatism/American College of Rheumatology criteria with SLEDAI. The 2019 criteria scores correlated positively with SLEDAI $(p<0.0001, r=0.4526)$. SLEDAI, SLE disease activity index.

noticed that, compared with the LS group, HS group was significantly higher in the both clinical and immunological scores (mucocutaneous including acute cutaneous lupus and non-scarring alopecia, serosal, musculoskeletal, haematological domains, anti-dsDNA antibodies and complements).

Furthermore, we wonder if class III/IV (proliferative) versus II/V (non-proliferative) groups differ on the EULAR/ACR scores after excluding the renal domains. It has been found that the 2019 criteria score of III/IV group were higher than that of II/V groups $(p=0.005)$ (online supplemental table 2). Specifically, the III/IV group differed significantly from the II/V group in terms of serosal, complement and specific autoantibodies domains $(\mathrm{p}<0.05)$.

\section{Comparison of renal histopathological findings in patients} with SLE with high and low criteria scores

The renal biopsy findings in the two groups are shown in table 2. The proportions of class III/IV and V LN for HS group were $71.19 \%$ and $13.56 \%$, respectively, and for LS group were $48.33 \%$ and $31.67 \%$, respectively $(\mathrm{p}=0.034)$. However, the prevalence of sclerotic glomeruli or crescents, the severity of mesangial hypercellularity or interstitial infiltrates and the existence of capillary necrosis showed no statistically significant difference between the two groups. The severity of glomerular immunofluorescence staining including IgA, IgM, IgG, C3, C4, C1q did not differ significantly between the HS group and the LS group. The AI index of patients with LN in HS group was significantly higher than that in LS group $(3.83 \pm 0.31$ vs $2.73 \pm 0.25, \mathrm{p}=0.007)$. However, the CI showed no difference between the two groups. Expectedly, compared with II/V group, the III/IV group increased severity in kidney histological findings (crescents, interstitial infiltrates and immunofluorescence microscopy of C3, C4 and C1q) and elevated AI and CI indexes $(\mathrm{p}<0.05)$ (online supplemental table 3).

Comparisons of renal function, treatments and renal outcomes in patients with SLE with high and low criteria scores

Theitems of renal function, treatments and renal outcomes are detailed in table 3 . The HS group displayed significantly more severe kidney damage, specifically the higher proportions of acute kidney injury, and lower estimated glomerular filtration rate (eGFR), whereas the HS group showed no significant differences in the variables of serum albumin level and uPCR. Compared with the LS group, the patients in the HS group received relatively higher proportions of intensive treatments such as methylprednisone impulses (38.98\% vs $24.59 \%)$, and temporary dialysis $(15.25 \%$ vs $3.28 \%, p=0.028)$. However, the patients in the HS group received lower proportions of merely prednisone in the induction treatment $(16.95 \%$ vs $31.15 \%)$ and the maintenance treatment $(18.64 \%$ vs $34.43 \%)$. Within the follow-up periods of $34.21 \pm 17.16$ months in HS group and $31.54 \pm 17.25$ months in LS group, the total of clinical renal remission rates, including complete remission and partial remission of the HS group and LS group were, respectively, $93.22 \%$ and $93.44 \%$. Of which, the rates of complete remission and partial remission were, respectively, $59.32 \%$ and $33.90 \%$ in the HS group, whereas were respectively, $68.85 \%$ and $24.59 \%$ in the LS group. The composite outcomes of ESRD and death were similar between patients in the HS and LS groups. The Kaplan-Meier curve by Cox regression analysis, adjusted for LN classification, eGFR, AI and CI indexes and induction and maintenance treatments, showed that patients in the HS group had a tendency of higher renal flare risk than that in the LS group (HR=0.206, 95\% CI 0.045 to $0.97, \mathrm{p}=0.04$ ) (figure 2 , online supplemental table 4).

\section{DISCUSSION}

These proposed EULAR/ACR criteria have strong operating characteristics using the structure and weighting in classification of SLE, ${ }^{5}$ promoting our understanding of the disease to help guide treatment strategies. Furthermore, the unique separation of renal biopsy findings addresses the importance of their differential impact on the SLE classification and underlying disease pathogenesis. ${ }^{17}{ }^{18}$ As a new tool for classification and research, the newly developed criteria would be undoubtedly useful in the clinical practice of nephrology clinic. Herein, we reported the first study to evaluate the performance of EULAR/ACR criteria for SLE in an Asian validation cohort of patients on the basis of the renal biopsy findings.

Our findings show that, due to the ANA entry requirement, the new criteria performed a sensitivity of $95 \%$ in our cohort were comparable with those of the 2012 SLICC criteria. Without their renal biopsies, the sensitivities of the new criteria were decreased from $95.24 \%$ to $89.68 \%$, which showed renal biopsy in patients help to 
Table 1 Comparison of clinical data between patients with SLE with high or low 2019 criteria score $(n=120)$

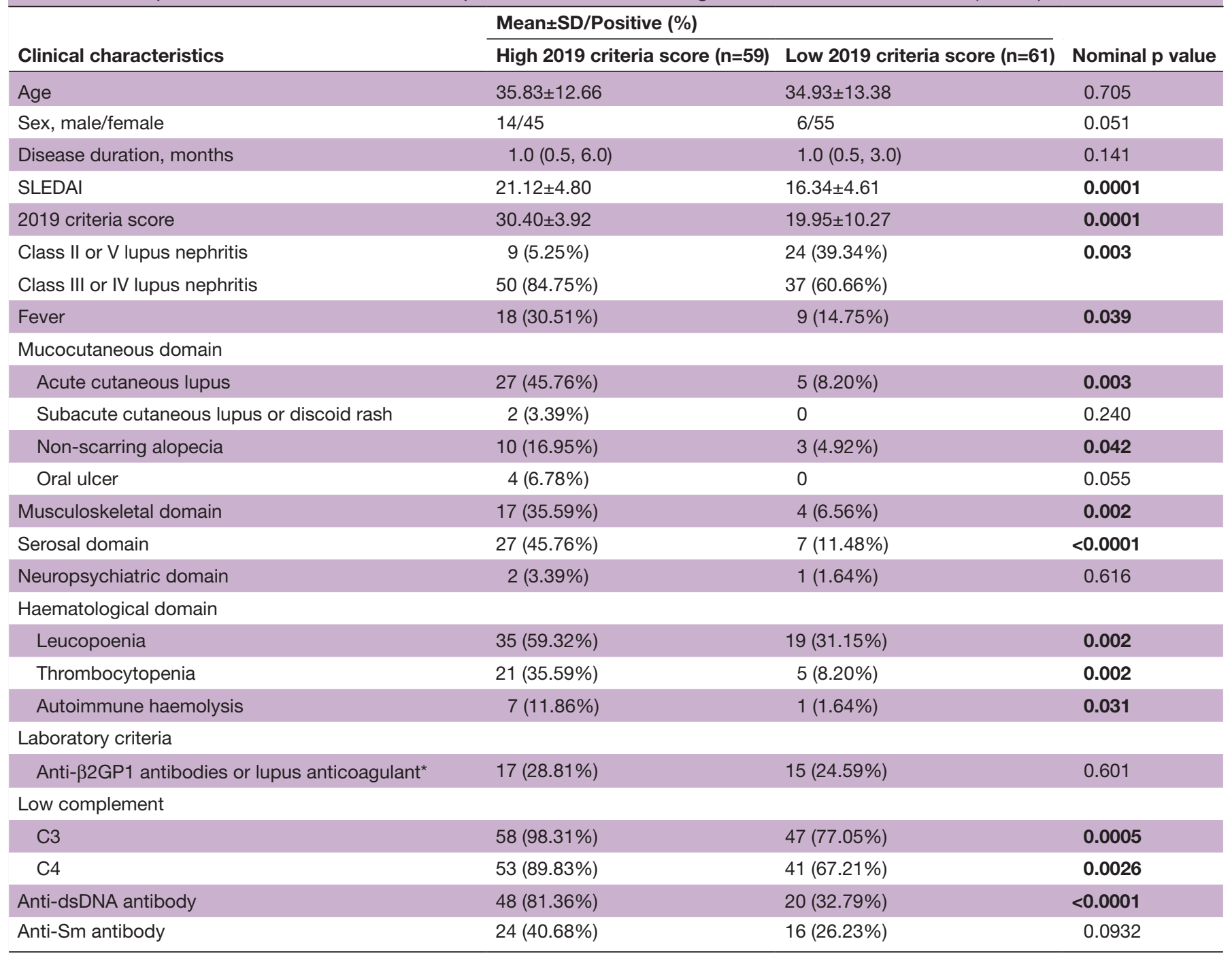

P-values $<0.05$ were considered as significance and marked in bold.

${ }^{*}$ The data of lupus anticoagulant were absent.

SLEDAI, SLE disease activity index.

optimise the classification of SLE. ${ }^{18}$ Similar to our results, the sensitivity of 2012 SLICC criteria is $100 \%$ in a cohort of patients with full house glomerular deposits. ${ }^{2}$ Additionally, it could be concluded that overall, the EULAR/ ACR classification performed well in our cohort, but the entry criterion of positive ANA $(\geq 1: 80)$ compromised the sensitivity, which is consistent with the recent findings. ${ }^{19}$ It has been reported that the 2019 criteria for SLE possibly misclassify the real SLE cases as non-SLE, especially if patients have a low titre $(<1: 80)$ of ANA. ${ }^{19}$ Notably, cut-off titres for mmunofluorescence (IF)-ANA differs among laboratories, depending on various factors. Consequently, it is doubtful whether a cut-off for IF-ANA of 1:80 is universal to use worldwide. ${ }^{20}$ This entry criterion has limited influence on increasing specificity of SLE classification. $^{21}$

In particular, the EULAR/ACR criteria score significantly correlated positively with the SLEDAI score in patients who fulfilled EULAR/ACR criteria in our cohort, which indicates that, to a certain extent, EULAR/ACR criteria can reflect the count that signifies severity of SLE. This result is in agreement with an SLE cohort without renal biopsy selected from rheumatology clinic. ${ }^{7}$ Moreover, compared with patients in the HS group, patients with SLE in the LS group had a significantly higher frequency of class II or V LN, a lower frequency of class III or IV LN, fever, acute cutaneous lupus, alopecia, joint involvement, serosal, neuropsychiatric and haematological manifestations, anti-dsDNA antibodies, low complements. Thus, patients with SLE in the HS group seemed to represent a phenotype of class III or IV LN with more extrarenal manifestations, which appears that the EULAR/ACR criteria could be used also as disease severity assessment and SLE is a complicated disease with heterogeneous phenotypes. ${ }^{22}$ In LUMINA cohort, they proposed, different from previous criteria, the 2019 EULAR/ACR could allow us to certify subsets of patients with different damage disease. ${ }^{23}$ Of interest, we observed 
Lupus Science \& Medicine

Table 2 Pathology findings in patients with SLE with high or low 2019 criteria score

\begin{tabular}{|c|c|c|c|}
\hline Finding & High 2019 criteria score $(n=59)$ & Low 2019 criteria score $(n=60)^{*}$ & Nominal $p$ value \\
\hline \multicolumn{4}{|l|}{ Light microscopy } \\
\hline \multicolumn{4}{|l|}{ ISN/RPS 2003 class } \\
\hline $\mathrm{III} / \mathrm{IV}$ & 42 (71.19\%) & $29(48.33 \%)$ & \\
\hline $\mathrm{III} / \mathrm{IV}+\mathrm{V}$ & $8(13.56 \%)$ & $8(13.33 \%)$ & \\
\hline v & $8(13.56 \%)$ & $19(31.67 \%)$ & \\
\hline Crescents, median (IQR), \% & $2.56(0,16.67)$ & $0(0,5.60)$ & 0.061 \\
\hline \multicolumn{4}{|l|}{ Capillary necrosis } \\
\hline Absent & $56(94.92 \%)$ & $54(90.0 \%)$ & 0.311 \\
\hline Existed & $3(5.08 \%)$ & $6(10.0 \%)$ & \\
\hline \multicolumn{4}{|l|}{ Mesangial hypercellularity } \\
\hline \multicolumn{4}{|l|}{ Interstitial infiltrates } \\
\hline $0 \sim 25 \%$ & $54(91.53 \%)$ & $52(86.67 \%)$ & 0.063 \\
\hline $25 \% \sim 50 \%$ & $5(8.47 \%)$ & $3(5.00 \%)$ & \\
\hline$>50 \%$ & 0 & $5(8.33 \%)$ & \\
\hline
\end{tabular}

Immunofluorescence microscopy

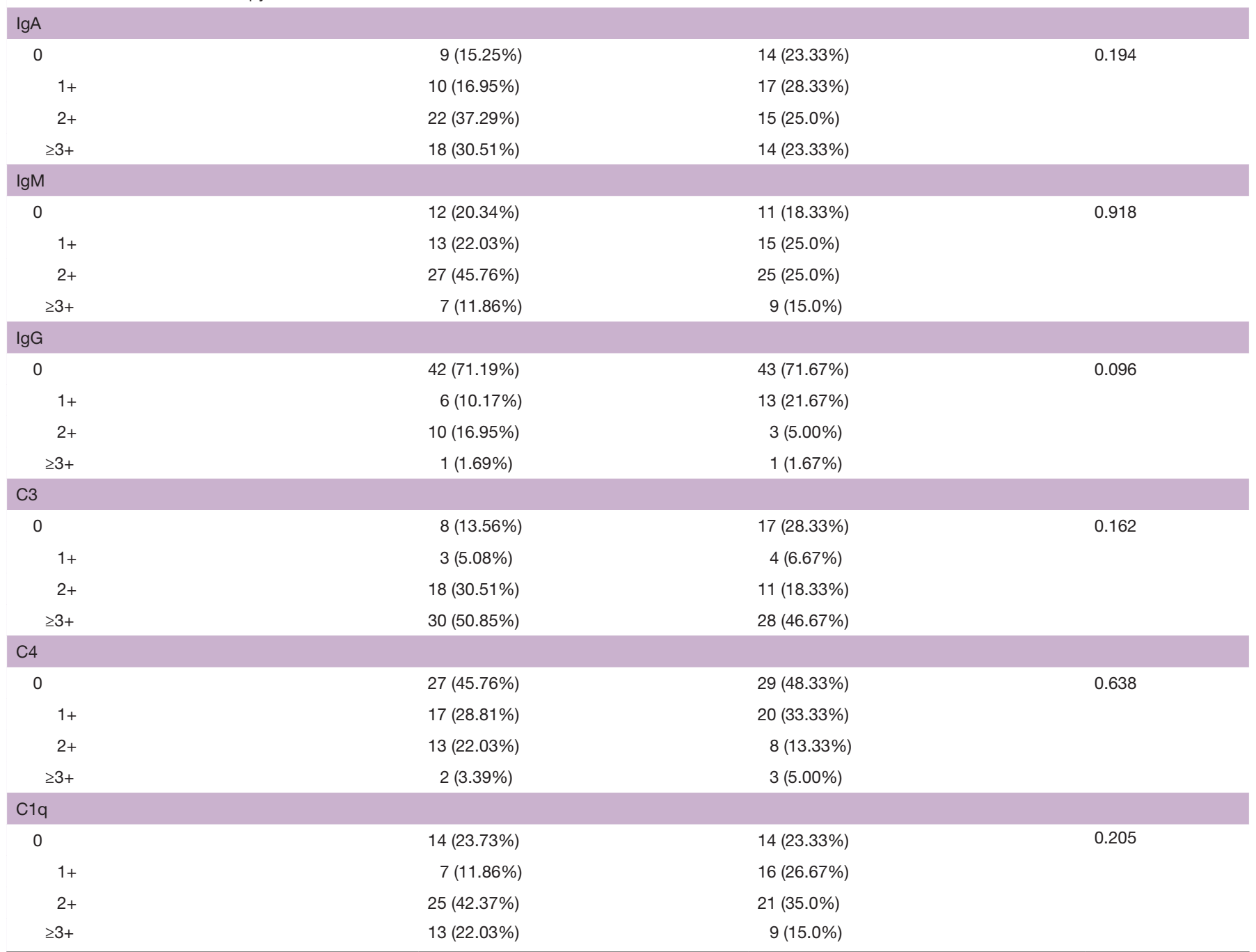

Continued 
Lupus nephritis

\begin{tabular}{lccc}
\hline Table 2 Continued & & & \\
\hline Finding & High 2019 criteria score $(\mathbf{n}=\mathbf{5 9})$ & Low $\mathbf{2 0 1 9}$ criteria score $(\mathbf{n}=\mathbf{6 0})^{*}$ & Nominal $\mathbf{p}$ value \\
\hline $\mathrm{Al}$ & $3.83 \pm 0.31$ & $2.73 \pm 0.25$ & $\mathbf{0 . 0 0 7}$ \\
$\mathrm{Cl}$ & $2.53 \pm 0.15$ & $2.77 \pm 0.21$ & 0.343 \\
\hline
\end{tabular}

P-values $<0.05$ were considered as significance and marked in bold.

${ }^{*}$ The data of pathology findings of one patient were absent.

$\mathrm{Al}$, activity index; $\mathrm{Cl}$, chronicity index.

the renal activity indices of HS group was significantly higher than that of LS group, which suggested patients with LN with high scores were more likely to accompany more severe LN. Concerning the renal pathological features, we found pathological findings, including light microscopy and immunofluorescene microscopy findings, were not significantly different between the HS group and LS group. It should note that patients with SLE of HS group with more proliferative LN showed higher percentage of crescents, which have previously been reported to be suggestive of severe active lesions in LN. ${ }^{14}{ }^{24}$ Overall, patients with LN with high new criteria score are more likely to suffer from proliferative LN.

Clinical studies suggested that renal function at baseline, renal flares and complete or partial remission were associated with long-term renal outcome in $\mathrm{LN} .{ }^{25-27}$ Interestingly, compared with those of the LS group, patients with SLE in the HS group showed more severe serological activity, including a markedly higher eGFR and a higher incidence of acute kidney injury. Consequently,

Table 3 Comparisons of renal-related indicators, therapeutic strategies and outcomes

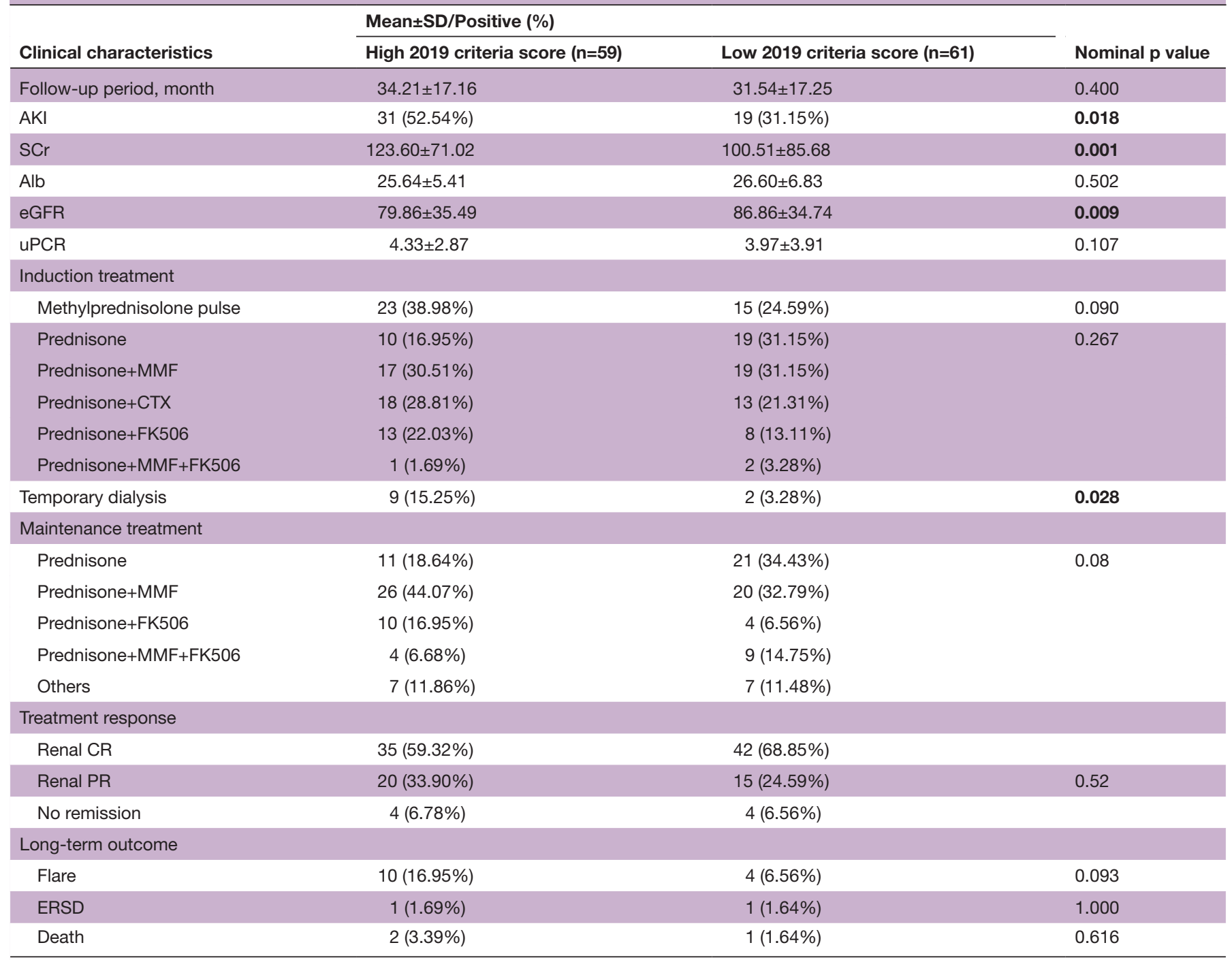

P-values $<0.05$ were considered as significance and marked in bold.

AKI, acute kidney injury; Alb, albumin; CR, complete remission; CTX, cyclophosphamide; eGFR, estimated glomerular filtration rate; ERSD, end-stage renal disease; MMF, mycophenolate mofetil; PR, partial remission; SCr, serum creatinine; uPCR, urine protein creatinine ratio. 


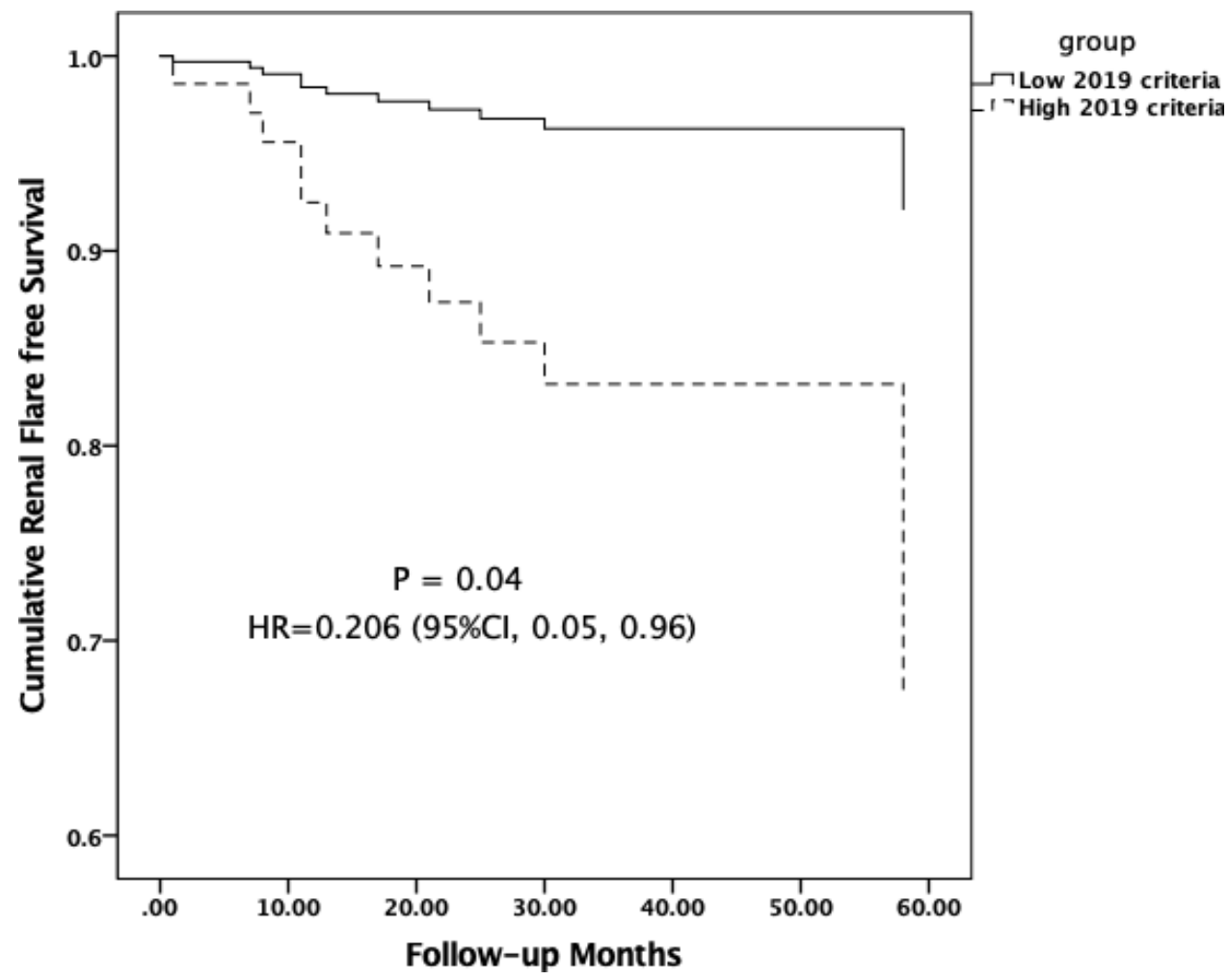

Figure 2 Comparisons of renal flare long term in patients with LN between the two groups. HS/LS: patients with LN with high/low 2019 European League Against Rheumatism/American College of Rheumatology criteria scores. The Kaplan-Meier exploratory analyses, adjusted for LN classification, estimated glomerular filtration rate, activity index and chronicity index, and induction and maintenance treatments, showed that patients in the HS group had a tendency of higher renal flare risk than that in the LS group ( $\mathrm{HR}=0.206,95 \% \mathrm{Cl} 0.045$ to $0.97, \mathrm{p}=0.04)$.

the short-term outcome showed no significant difference between the two groups. After the similar duration follow up of the disease, the results herein showed a trend, that compared with the patients in the LS group, patients with SLE in the HS group appeared to have a higher proportion of renal flares. It is noteworthy that in our cohort, patients with high new criteria score are indeed at high risk of renal flares and need proper therapeutic strategy, which supports the utility of EULAR/ACR classification in everyday clinical practice.

These findings represent additional validation for the recently proposed EULAR/ACR criteria in a specific patient cohort. There are several limitations in this study. First, our current study is a retrospective study with a relatively small size of samples and the lack of the data of lupus anticoagulant in only single centre. In a real-world setting, we were not able to routinely measure all parameters included in the 2019 criteria. Second, our study population contained subjects with confirmed LN cases and a reasonable suspicion of SLE referred to only nephrology specialists rather than rheumatology physicians. Third, we assessed the performance of EULAR/ACR criteria by merely one cut-off of new criteria in our cohort. We need to amplify individuals with renal biopsy and use several cut-offs of new criteria in a multicentre cohort to validate our observations in the future.

In conclusion, our study showed that EULAR/ACR classification proven to have great sensitivity among patients with SLE with a renal biopsy and highlights the potential impact of the proposed EULAR/ACR criteria. Our results also supported its use in clinical care and research, which can prevent some of these patients from qualifying for future clinical trials for SLE.

Acknowledgements The authors would like to thank all the clinicians at Kidney Disease Centre, the First Affiliated Hospital, College of Medicine, Zhejiang University, for their efforts in this study.

Contributors All authors were involved in analysing and interpreting the data, drafting the article and revising it critically for important intellectual content, and all read and approved the final version to be published.

Funding This study was supported by the funds from Primary Research \& Development plan of Zhejiang Province (2020C03034) and the key research project of precision medicine in National Key Research and Development Plan (2017YFC0907603) to FH, Zhejiang Medical and Health Science and Technology Project (2019RC036) to $L L$ and Project of Natural Science Foundation of Zhejiang Province (Q19H050030) to PR.

Competing interests None declared.

Patient consent for publication Not required.

Ethics approval This study protocol was approved by the Ethics Committee of the First Affiliated Hospital of Zhejiang University School of Medicine (No. 201745). Informed consents were not obtained from the patients for this study, as approved by the Ethics Committee.

Provenance and peer review Not commissioned; externally peer reviewed. Data availability statement Data are available upon reasonable request to the corresponding author (hanf8876@zju.edu.cn).

Supplemental material This content has been supplied by the author(s). It has not been vetted by BMJ Publishing Group Limited (BMJ) and may not have been peer-reviewed. Any opinions or recommendations discussed are solely those of the author(s) and are not endorsed by BMJ. BMJ disclaims all liability and 
responsibility arising from any reliance placed on the content. Where the content includes any translated material, BMJ does not warrant the accuracy and reliability of the translations (including but not limited to local regulations, clinical guidelines, terminology, drug names and drug dosages), and is not responsible for any error and/or omissions arising from translation and adaptation or otherwise.

Open access This is an open access article distributed in accordance with the Creative Commons Attribution Non Commercial (CC BY-NC 4.0) license, which permits others to distribute, remix, adapt, build upon this work non-commercially, and license their derivative works on different terms, provided the original work is properly cited, appropriate credit is given, any changes made indicated, and the use is non-commercial. See: http://creativecommons.org/licenses/by-nc/4.0/.

ORCID iD

Fei Han http://orcid.org/0000-0001-5363-7556

\section{REFERENCES}

1 Lisnevskaia L, Murphy G, Isenberg D. Systemic lupus erythematosus. Lancet 2014;384:1878-88.

2 Rijnink EC, Teng YKO, Kraaij T, et al. Validation of the systemic lupus international collaborating clinics classification criteria in a cohort of patients with full house glomerular deposits. Kidney Int 2018;93:214-20.

3 Petri M, Orbai A-M, Alarcón GS, et al. Derivation and validation of the systemic lupus international collaborating clinics classification criteria for systemic lupus erythematosus. Arthritis Rheum 2012;64:2677-86.

4 Hartman EAR, van Royen-Kerkhof A, Jacobs JWG, et al. Performance of the 2012 systemic lupus international collaborating clinics classification criteria versus the 1997 American College of rheumatology classification criteria in adult and juvenile systemic lupus erythematosus. A systematic review and meta-analysis. Autoimmun Rev 2018;17:316-22.

5 Aringer M, Costenbader K, Daikh D, et al. 2019 European League against Rheumatism/American College of rheumatology classification criteria for systemic lupus erythematosus. Ann Rheum Dis 2019;78:1151-9.

6 Aringer M, Costenbader K, Daikh D, et al. 2019 European League against Rheumatism/American College of rheumatology classification criteria for systemic lupus erythematosus. Arthritis Rheumatol 2019;71:1400-12.

7 Teng J, Zhou Z, Wang F, et al. Do 2019 European League against rheumatism/American College of rheumatology classification criteria for systemic lupus erythematosus also indicate the disease activity? Ann Rheum Dis 2020:annrheumdis-2020-217017.

8 Gladman DD, Ibañez D, Urowitz MB. Systemic lupus erythematosus disease activity index 2000. J Rheumatol 2002;29:288-91.

9 Weening JJ, D'Agati VD, Schwartz MM, et al. The classification of glomerulonephritis in systemic lupus erythematosus revisited. Kidney Int 2004;65:521-30.

10 Ginzler EM, Dooley MA, Aranow C, et al. Mycophenolate mofetil or intravenous cyclophosphamide for lupus nephritis. N Engl J Med 2005;353:2219-28.
11 Yu F, Tan Y, Liu G, et al. Clinicopathological characteristics and outcomes of patients with crescentic lupus nephritis. Kidney Int 2009;76:307-17.

12 Chapter 12: lupus nephritis. Kidney Int Supp/ 2012;2:221-32.

13 Chen S, Chen H, Liu Z, et al. Pathological spectrums and renal prognosis of severe lupus patients with rapidly progressive glomerulonephritis. Rheumatol Int 2015;35:709-17.

14 Cai F, Han F, Wang H, et al. The crescentic implication of renal outcomes in proliferative lupus nephritis. $J$ Rheumatol 2018;45:513-20.

15 Floege J, Barbour SJ, Cattran DC, et al. Management and treatment of glomerular diseases (Part 1): conclusions from a kidney disease: improving global outcomes (KDIGO) controversies conference. Kidney Int 2019;95:268-80.

16 Rubio J, Krishfield S, Kyttaris VC. Application of the 2019 European League against Rheumatism/American College of rheumatology systemic lupus erythematosus classification criteria in clinical practice: a single center experience. Lupus 2020;29:421-5.

17 De Rosa M, Azzato F, Toblli JE, et al. A prospective observational cohort study highlights kidney biopsy findings of lupus nephritis patients in remission who flare following withdrawal of maintenance therapy. Kidney Int 2018;94:788-94.

18 Yu F, Haas M, Glassock R, et al. Redefining lupus nephritis: clinical implications of pathophysiologic subtypes. Nat Rev Nephrol 2017;13:483-95.

19 Suda M, Kishimoto M, Ohde S, et al. Validation of the 2019 ACR/ EULAR classification criteria of systemic lupus erythematosus in 100 Japanese patients: a real-world setting analysis. Clin Rheumatol 2020;39:1823-7.

20 Infantino M, Manfredi M, Bizzaro N, et al. European League against Rheumatism/American College of rheumatology classification criteria for systemic lupus erythematosus: the laboratory Immunologist's point of view. Ann Rheum Dis 2019:annrheumdis-2019-216591.

21 Aringer M, Costenbader KH, Dörner T, et al. Limited role of specificity of ANA for SLE classification. Response to: 'European League against Rheumatism/American College of Rheumatology classification criteria for systemic lupus erythematosus: the laboratory immunologist's point of view' by Infantino et al. Ann Rheum Dis 2019:annrheumdis-2019-216700.

22 Dörner T, Furie R. Novel paradigms in systemic lupus erythematosus. Lancet 2019;393:2344-58.

23 Ugarte-Gil MF, Pons-Estel GJ, Griffin R, et al. Patients who do not fulfill the 2019 EULAR/ACR criteria for systemic lupus erythematosus Accrue less damage. Arthritis Care Res 2020.

24 Rijnink EC, Teng YKO, Wilhelmus S, et al. Clinical and histopathologic characteristics associated with renal outcomes in lupus nephritis. Clin J Am Soc Nephrol 2017;12:734-43.

25 Chrysochou C, Randhawa H, Reeve R, et al. Determinants of renal functional outcome in lupus nephritis: a single centre retrospective study. QJM 2008;101:313-6.

26 Chen YE, Korbet SM, Katz RS, et al. Value of a complete or partial remission in severe lupus nephritis. Clin J Am Soc Nephrol 2008:3:46-53.

27 Mejía-Vilet JM, Córdova-Sánchez BM, Arreola-Guerra JM, et al. Renal flare prediction and prognosis in lupus nephritis Hispanic patients. Lupus 2016;25:315-24. 